\title{
An analysis of cross-continental scholarship requirements during neurosurgical training and national research productivity
}

\author{
Michael S. Rallo, BS, Omar Ashraf, BA, Fareed Jumah, MD, Gaurav Gupta, MD, and \\ Anil Nanda, MD, MPH \\ Department of Neurosurgery, Rutgers Robert Wood Johnson Medical School, New Brunswick, New Jersey
}

OBJECTIVE Engagement in research and scholarship is considered a hallmark of neurosurgical training. However, the participation of neurosurgical trainees in this experience has only recently been analyzed and described in the United States, with little, if any, data available regarding the research environment in neurosurgical training programs across the globe. Here, the authors set out to identify requirements for research involvement and to quantify publication rates in leading neurosurgical journals throughout various nations across the globe.

METHODS The first aim was to identify the research requirements set by relevant program-accrediting and/or boardcertifying agencies via query of the literature and published guidelines. For the second part of the study, the authors attempted to determine each country's neurosurgical research productivity by quantifying publications in the various large international neurosurgical journals-World Neurosurgery, Journal of Neurosurgery, and Neurosurgery-via a structured search of PubMed.

RESULTS Data on neurosurgical training requirements addressing research were available for $54(28.1 \%)$ of 192 countries. Specific research requirements were identified for 39 countries, partial requirements for 8 , and no requirements for 7. Surprisingly, the authors observed a trend of increased average research productivity with the absence of designated research requirements, although this finding is not unprecedented in the literature.

CONCLUSIONS A variety of countries of various sizes and neurosurgical workforce densities across the globe have instituted research requirements during training and/or prior to board certification in neurosurgery. These requirements range in intensity from 1 publication or presentation to the completion of a thesis or dissertation and occur at various time points throughout training. While these requirements do not correlate directly to national research productivity, they may provide a foundation for developing countries to establish a culture of excellence in research.

https://thejns.org/doi/abs/10.3171/2019.12.FOCUS19856

KEYWORDS neurosurgical trainee research; global neurosurgery; neurosurgery education

$\mathrm{E}$ NGAGEMENT in research and scholarship is considered a hallmark of neurosurgical training. This academic pursuit is fundamental to the advancement of the field. However, the participation of neurosurgical trainees in this experience has only recently been analyzed and described in the United States, ${ }^{18,19}$ with little, if any, data available regarding the research environment in neurosurgical training programs across the globe.

Throughout the United States, participation in research during neurosurgical training is nearly universal, despite a lack of explicit requirements set by residency-accrediting organizations and board-certifying agencies. ${ }^{1,3,19} \mathrm{~A}$ recent analysis of 1506 residents in 117 distinct neurosurgical training programs identified the average productivity of individual residents as 5 publications in total, with 3 published during residency.$^{19}$ Moreover, one's involvement in neurosurgical research typically begins early, with 1styear residents averaging at least 1 publication prior to the start of residency. ${ }^{18}$ This is likely attributable to both the academic nature of neurosurgery as a specialty and the high value placed on applicant involvement in research by residency program directors. Indeed, according to data

ABBREVIATIONS COSECSA = College of Surgeons of East, Central and Southern Africa; JRAAC = Joint Residency Advisory and Accreditation Committee; WFNS = World Federation of Neurosurgical Societies.

SUBMITTED November 1, 2019. ACCEPTED December 17, 2019.

INCLUDE WHEN CITING DOI: 10.3171/2019.12.FOCUS19856. 
presented by the National Resident Match Program, neurological surgery ranked as the highest in mean number of abstracts, presentations, and publications among allopathic seniors who matched into the specialty in $2018 .{ }^{24}$ One of the most significant factors in determining the productivity of these trainees is the extent of support offered by their institutions. Institutions can foster supporting educational environments via the provision of protected research time, funding, medical editorial assistance, and travel stipends..$^{19}$ In fact, the lack of protected time and institutional resources, including training in research design and conduct, has been identified as one of the greatest barriers to research involvement among physicians in various specialties..$^{9,21}$ Notably, however, a program's designation of a "research requirement" was not shown to have a significant impact on departmental productivity in the large neurosurgery resident cohort mentioned above. Authors of that study reasoned that research is engrained in the culture of many of the highly productive programs and does not require formal designation, while programs with an explicit requirement may be in the early stages of such a culture change..$^{19}$

The translation of these findings to the global arena of neurosurgery is unclear and likely influenced by various country- or region-specific factors including socioeconomic status, density of neurosurgeons, and research infrastructure and support. At present, there is limited literature on identifying and evaluating research requirements during neurosurgical training in nations outside of North America. In an important study focused on identifying requirements for neurosurgery certification across the globe, Gasco et al. reported that $3.8 \%$ of responding international societies require involvement in a research project, with publication, prior to oral examination eligibility. ${ }^{15}$ However, this analysis was limited to member societies of the World Federation of Neurosurgical Societies (WFNS) and did not explore in depth the impact of the requirement, nor other factors on research productivity in the responding countries. In appreciation of the great value of participation in the pursuit of knowledge via research and/or scholarship, our group has set out to identify research requirements established by relevant accrediting and/or certifying bodies across the globe. Additionally, we seek to understand how these requirements correspond to productivity.

\section{Methods \\ Data Extraction}

A list of countries was compiled from the WFNS Global Neurosurgical Workforce Map (https://www.wfns.org/ menu/61/global-neurosurgical-workforce-map). Our first aim was to identify research requirements set by relevant program-accrediting and/or board-certifying agencies within each country. This search was conducted using a general query of Google and the PubMed database to obtain the above described information from agency/society guidelines and previously reported literature. Search terms for these queries included various combinations of the country name AND "neurosurgery" AND "residency" OR "training" OR "education" AND "research."
These queries returned results including websites of national/regional neurosurgical certification organizations and previously published scholarly literature, which facilitated our determination of research requirements during training. Required research was defined as any compulsory research activity during residency, such as mandatory publications, presentations, thesis/dissertation, or research time. Explicit requirements including number of publications and/or presentations, when listed, were recorded for analysis. Undefined scholarly requirements were excluded from our analysis.

For the second part of the study, we aimed to determine each country's neurosurgical research productivity, which was assessed by quantifying a given country's publications in the various large international neurosurgical journals: World Neurosurgery, Journal of Neurosurgery, and Neurosurgery. These data were collected by performing a query of the PubMed database using the name of each country as the term in the "affiliation" search field in combination with the title of each journal in the "journal" search field. Our final PubMed query was as follows: "<journal>"[Journal] AND "<country>"[Affiliation] with no restriction on time.

\section{Data Analysis}

We performed a descriptive analysis of the research requirements and productivity across the countries for which data were available. These countries were grouped into 3 categories based on the nature of their requirements: 1) no stated requirement; 2) partial requirement, defined as a guideline lacking explicit details and/or only requiring education on research methodology; and 3) specific requirement, defined as a guideline with details on the type of research required and/or number of publications/ presentations. The number of publications for countries within each group was averaged, with descriptive statistics reported as the mean and range.

\section{Results}

We identified a total of 192 countries from the WFNS Global Neurosurgery Workforce Map. A complete list of countries and research output is included in Table 1, which displays each country along with information regarding that country's continent and geographic region as well as its number of publications in the aforementioned journals. Data regarding neurosurgical training requirements for research were available for $54(28.1 \%)$ of the 192 countries. Specific research requirements, as defined above, were identified for 39 countries, partial requirements for 8 , and no requirements for 7 (Table 2). The most frequently occurring reporting body was the College of Surgeons of East, Central and Southern Africa (COSECSA), which oversees surgical training in 14 African countries. In Europe, such data were primarily reported by the Joint Residency Advisory and Accreditation Committee (JRAAC), which is an agency that accredits residency programs throughout the various countries in the European Union. Finally, requirements in several nations were set by board-certifying agencies, such as the Japan Neurosurgical Society, making research obligatory in order to be board certified, although 
TABLE 1. Summary of countries, with information regarding their geographic location and research output in three neurosurgical journals

\begin{tabular}{|c|c|c|c|c|c|}
\hline \multirow[b]{2}{*}{ Country } & \multirow[b]{2}{*}{ Continent } & \multirow[b]{2}{*}{ Region } & \multicolumn{3}{|c|}{ No. of Publications } \\
\hline & & & World Neurosurg & Neurosurg & J Neurosurg \\
\hline Afghanistan & Asia & South Asia & 0 & 0 & 0 \\
\hline Albania & Europe & Balkan Peninsula & 4 & 0 & 3 \\
\hline Algeria & Africa & North Africa & 3 & 0 & 1 \\
\hline Angola & Africa & South-Central Africa & 1 & 0 & 0 \\
\hline Antigua \& Barbuda & North America & Caribbean & 0 & 0 & 0 \\
\hline Argentina & South America & Southern South America & 37 & 19 & 21 \\
\hline Armenia & Eurasia & Caucasia & 1 & 0 & 0 \\
\hline Australia & Oceania & & 140 & 140 & 117 \\
\hline Austria & Europe & Central Europe & 75 & 65 & 81 \\
\hline Azerbaijan & Eurasia & Caucasia & 1 & 0 & 0 \\
\hline Bahamas & North America & Caribbean & 0 & 0 & 0 \\
\hline Bahrain & Asia & Gulf & 0 & 0 & 0 \\
\hline Bangladesh & Asia & South Asia & 5 & 0 & 1 \\
\hline Barbados & North America & Caribbean & 0 & 0 & 0 \\
\hline Belarus & Europe & Eastern Europe & 0 & 0 & 1 \\
\hline Belgium & Europe & Western Europe & 65 & 100 & 75 \\
\hline Belize & North America & Central America & 0 & 0 & 0 \\
\hline Benin & Africa & West Africa & 2 & 0 & 0 \\
\hline Bhutan & Asia & South Asia & 0 & 0 & 0 \\
\hline Bosnia \& Herzegovina & Europe & Balkan Peninsula & 5 & 0 & 0 \\
\hline Botswana & Africa & South Africa & 0 & 0 & 0 \\
\hline Brazil & South America & & 234 & 110 & 103 \\
\hline Brunei Darussalam & Asia & Maritime Southeast Asia & 0 & 0 & 0 \\
\hline Bulgaria & Europe & Southeast Europe & 4 & 4 & 0 \\
\hline Burkina Faso & Africa & West Africa & 1 & 0 & 0 \\
\hline Burundi & Africa & East Africa & 0 & 0 & 0 \\
\hline Cabo Verde & Africa & West Africa & 0 & 0 & 0 \\
\hline Cambodia & Asia & Southeast Asia & 10 & 0 & 0 \\
\hline Cameroon & Africa & Central Africa & 0 & 0 & 0 \\
\hline Canada & North America & & 342 & 570 & 682 \\
\hline Cayman Islands & North America & Caribbean & 0 & 0 & 0 \\
\hline Central African Republic & Africa & Central (Middle) Africa & 0 & 0 & 0 \\
\hline Chad & Africa & North-Central Africa & 0 & 0 & 0 \\
\hline Chile & South America & Western South America & 35 & 8 & 0 \\
\hline China & Asia & East Asia & 1946 & 235 & 336 \\
\hline Colombia & South America & Northern South America & 35 & 14 & 8 \\
\hline Comoros & Africa & East Africa & 0 & 0 & 0 \\
\hline Costa Rica & North America & Central America & 0 & 1 & 0 \\
\hline Côte d'Ivoire & Africa & West Africa & 0 & 0 & 1 \\
\hline Croatia & Europe & Balkan Peninsula & 12 & 3 & 8 \\
\hline Cuba & North America & Caribbean & 2 & 1 & 0 \\
\hline Cyprus & Europe & Mediterranean Basin & 4 & 0 & 0 \\
\hline Czech Republic & Europe & Central Europe & 20 & 16 & 33 \\
\hline Democratic People's Republic of Korea & Asia & Korean Peninsula & 1 & 0 & 0 \\
\hline Democratic Republic of the Congo & Africa & Central Africa & 0 & 0 & 0 \\
\hline Denmark & Europe & Scandinavia & 25 & 31 & 36 \\
\hline Dominica & North America & Caribbean & 1 & 0 & 0 \\
\hline
\end{tabular}


» CONTINUED FROM PAGE 3

TABLE 1. Summary of countries, with information regarding their geographic location and research output in three neurosurgical journals

\begin{tabular}{|c|c|c|c|c|c|}
\hline \multirow[b]{2}{*}{ Country } & \multirow[b]{2}{*}{ Continent } & \multirow[b]{2}{*}{ Region } & \multicolumn{3}{|c|}{ No. of Publications } \\
\hline & & & World Neurosurg & Neurosurg & J Neurosurg \\
\hline Dominican Republic & North America & Caribbean & 2 & 0 & 2 \\
\hline Ecuador & South America & Western South America & 3 & 0 & 0 \\
\hline Egypt & Africa & Northeast Africa & 92 & 19 & 34 \\
\hline El Salvador & North America & Central America & 0 & 0 & 0 \\
\hline Equatorial Guinea & Africa & Central (Middle) Africa & 0 & 0 & 0 \\
\hline Eritrea & Africa & East Africa & 0 & 0 & 0 \\
\hline Estonia & Europe & Northern Europe & 0 & 1 & 1 \\
\hline eSwatini & Africa & South Africa & 0 & 0 & 0 \\
\hline Ethiopia & Africa & East Africa & 13 & 0 & 0 \\
\hline Federated States of Micronesia & Oceania & Micronesia & 0 & 0 & 0 \\
\hline Fiji & Oceania & Melanesia & 0 & 0 & 0 \\
\hline Finland & Europe & Northern Europe & 102 & 106 & 53 \\
\hline France & Europe & & 273 & 365 & 366 \\
\hline Gabon & Africa & West-Central Africa & 0 & 0 & 0 \\
\hline Georgia & Eurasia & Caucasia & 0 & 0 & 0 \\
\hline Germany & Europe & Western Europe & 586 & 754 & 644 \\
\hline Ghana & Africa & West Africa & 4 & 0 & 0 \\
\hline Greece & Europe & Balkan Peninsula & 71 & 61 & 61 \\
\hline Grenada & North America & Caribbean & 58 & 7 & 5 \\
\hline Guatemala & North America & Central America & 1 & 0 & 0 \\
\hline Guinea & Africa & West Africa & 0 & 0 & 0 \\
\hline Guinea-Bissau & Africa & West Africa & 0 & 0 & 0 \\
\hline Guyana & South America & Northern South America & 0 & 0 & 0 \\
\hline Haiti & North America & Caribbean & 5 & 0 & 1 \\
\hline Honduras & North America & Central America & 3 & 0 & 0 \\
\hline Hungary & Europe & Central Europe & 17 & 14 & 19 \\
\hline Iceland & Europe & North Atlantic & 1 & 1 & 0 \\
\hline India & Asia & South Asia & 448 & 121 & 136 \\
\hline Indonesia & Asia & Maritime Southeast Asia & 12 & 0 & 4 \\
\hline Iran & Asia & West Asia & 122 & 22 & 36 \\
\hline Iraq & Asia & West Asia & 6 & 1 & 0 \\
\hline Ireland & Europe & British Isles & 22 & 19 & 14 \\
\hline Israel & Asia & West Asia & 149 & 123 & 124 \\
\hline Italy & Europe & Southern Europe & 698 & 432 & 303 \\
\hline Jamaica & North America & Caribbean & 0 & 0 & 0 \\
\hline Japan & Asia & Eastern Asia & 1108 & 1230 & 1685 \\
\hline Jordan & Asia & Western Asia & 7 & 2 & 2 \\
\hline Kazakhstan & Asia & Central Asia & 3 & 0 & 1 \\
\hline Kenya & Africa & East Africa & 15 & 0 & 0 \\
\hline Kiribati & Oceania & Micronesia & 0 & 0 & 0 \\
\hline Kosovo & Europe & Balkan Peninsula & 0 & 0 & 0 \\
\hline Kuwait & Asia & Arabian Peninsula & 8 & 2 & 2 \\
\hline Kyrgyzstan & Asia & Central Asia & 0 & 0 & 0 \\
\hline Lao People's Democratic Republic & Asia & Mainland Southeast Asia & 0 & 0 & 0 \\
\hline Latvia & Europe & Northern Europe & 0 & 0 & 0 \\
\hline Lebanon & Asia & Western Asia & 48 & 56 & 47 \\
\hline
\end{tabular}


» CONTINUED FROM PAGE 4

TABLE 1. Summary of countries, with information regarding their geographic location and research output in three neurosurgical journals

\begin{tabular}{|c|c|c|c|c|c|}
\hline \multirow[b]{2}{*}{ Country } & \multirow[b]{2}{*}{ Continent } & \multirow[b]{2}{*}{ Region } & \multicolumn{3}{|c|}{ No. of Publications } \\
\hline & & & World Neurosurg & Neurosurg & J Neurosurg \\
\hline Lesotho & Africa & South Africa & 0 & 0 & 0 \\
\hline Liberia & Africa & West Africa & 0 & 0 & 0 \\
\hline Libya & Africa & North Africa & 0 & 1 & 0 \\
\hline Lithuania & Europe & Northern Europe & 4 & 2 & 1 \\
\hline Luxembourg & Europe & Western Europe & 2 & 0 & 0 \\
\hline Madagascar & Africa & East Africa & 0 & 0 & 0 \\
\hline Malawi & Africa & Southeast Africa & 2 & 0 & 0 \\
\hline Malaysia & Asia & Maritime Southeast Asia & 10 & 4 & 4 \\
\hline Maldives & Asia & South Asia & 0 & 0 & 0 \\
\hline Mali & Africa & West Africa & 0 & 0 & 0 \\
\hline Malta & Europe & Mediterranean Basin & 0 & 0 & 0 \\
\hline Marshall Islands & Oceania & Micronesia & 0 & 0 & 0 \\
\hline Martinique & North America & Caribbean & 0 & 0 & 0 \\
\hline Mauritania & Africa & Northwest Africa & 0 & 0 & 0 \\
\hline Mauritius & Africa & East Africa & 0 & 0 & 0 \\
\hline Mexico & North America & & 104 & 61 & 58 \\
\hline Mongolia & Asia & East Asia & 3 & 0 & 1 \\
\hline Montenegro & Europe & Southeast Europe & 0 & 2 & 1 \\
\hline Morocco & Africa & North Africa & 22 & 6 & 3 \\
\hline Mozambique & Africa & Southeast Africa & 2 & 0 & 0 \\
\hline Myanmar & Asia & Mainland Southeast Asia & 3 & 0 & 0 \\
\hline Namibia & Africa & South Africa & 0 & 0 & 0 \\
\hline Nauru & Oceania & Micronesia & 0 & 0 & 0 \\
\hline Nepal & Asia & South Asia & 20 & 0 & 1 \\
\hline New Zealand & Oceania & Polynesia & 18 & 14 & 9 \\
\hline Nicaragua & North America & Central America & 4 & 0 & 0 \\
\hline Niger & Africa & West Africa & 0 & 0 & 0 \\
\hline Nigeria & Africa & West Africa & 24 & 2 & 5 \\
\hline North Macedonia & Europe & Balkan Peninsula & 0 & 4 & 0 \\
\hline Norway & Europe & Scandinavian Peninsula & 55 & 84 & 61 \\
\hline Oman & Asia & Arabian Peninsula & 2 & 0 & 1 \\
\hline Pakistan & Asia & South Asia & 35 & 3 & 2 \\
\hline Palau & Oceania & Micronesia & 0 & 0 & 0 \\
\hline Panama & North America & Central America & 2 & 0 & 0 \\
\hline Papua New Guinea & Oceania & Melanesia & 1 & 0 & 0 \\
\hline Paraguay & South America & Central South America & 0 & 0 & 0 \\
\hline Peru & South America & Western South America & 5 & 0 & 1 \\
\hline Philippines & Asia & Maritime Southeast Asia & 9 & 0 & 7 \\
\hline Plurinational State of Bolivia & South America & Western South America & 4 & 0 & 1 \\
\hline Poland & Europe & Central Europe & 36 & 9 & 16 \\
\hline Portugal & Europe & Iberian Peninsula & 17 & 15 & 7 \\
\hline Puerto Rico & North America & Caribbean & 10 & 7 & 15 \\
\hline Qatar & Asia & Arabian Peninsula & 5 & 0 & 2 \\
\hline Republic of Korea & Asia & Korean Peninsula & 252 & 75 & 100 \\
\hline Republic of Moldova & Europe & Eastern Europe & 0 & 0 & 0 \\
\hline Republic of the Congo & Africa & Central Africa & 0 & 0 & 0 \\
\hline
\end{tabular}


» CONTINUED FROM PAGE 5

TABLE 1. Summary of countries, with information regarding their geographic location and research output in three neurosurgical journals

\begin{tabular}{|c|c|c|c|c|c|}
\hline \multirow[b]{2}{*}{ Country } & \multirow[b]{2}{*}{ Continent } & \multirow[b]{2}{*}{ Region } & \multicolumn{3}{|c|}{ No. of Publications } \\
\hline & & & World Neurosurg & Neurosurg & J Neurosurg \\
\hline Romania & Europe & Balkan Peninsula & 18 & 2 & 5 \\
\hline Russian Federation & Europe & Eastern Europe & 61 & 5 & 14 \\
\hline Rwanda & Africa & Central Africa & 6 & 0 & 0 \\
\hline Saint Kitts \& Nevis & North America & Caribbean & 0 & 0 & 0 \\
\hline Saint Lucia & North America & Caribbean & 0 & 0 & 0 \\
\hline Samoa & Oceania & Polynesia & 0 & 0 & 0 \\
\hline São Tomé and Príncipe & Africa & West Africa & 0 & 0 & 0 \\
\hline Saudi Arabia & Asia & Arabian Peninsula & 71 & 15 & 22 \\
\hline Senegal & Africa & West Africa & 1 & 0 & 0 \\
\hline Serbia & Europe & Balkan Peninsula & 15 & 8 & 3 \\
\hline Seychelles & Africa & East Africa & 0 & 0 & 0 \\
\hline Sierra Leone & Africa & West Africa & 0 & 0 & 0 \\
\hline Singapore & Asia & Maritime Southeast Asia & 32 & 26 & 22 \\
\hline Slovakia & Europe & Central Europe & 4 & 0 & 3 \\
\hline Slovenia & Europe & Southern Central Europe & 0 & 7 & 7 \\
\hline Solomon Islands & Oceania & Melanesia & 0 & 0 & 0 \\
\hline Somalia & Africa & East Africa & 0 & 0 & 0 \\
\hline South Africa & Africa & South Africa & 27 & 30 & 21 \\
\hline South Sudan & Africa & East-Central Africa & 0 & 0 & 0 \\
\hline Spain & Europe & Southwestern Europe & 169 & 110 & 144 \\
\hline Sri Lanka & Asia & South Asia & 0 & 0 & 0 \\
\hline State of Palestine & Asia & Western Asia & 13 & 0 & 0 \\
\hline Sudan & Africa & Northeast Africa & 3 & 0 & 0 \\
\hline Suriname & South America & Northeastern South America & 0 & 0 & 0 \\
\hline Sweden & Europe & Scandinavian Peninsula & 53 & 135 & 149 \\
\hline Switzerland & Europe & Central Europe & 190 & 134 & 140 \\
\hline Syrian Arab Republic & Asia & West Asia & 1 & 0 & 0 \\
\hline Tajikistan & Asia & Central Asia & 0 & 0 & 0 \\
\hline Tanzania & Africa & East Africa & 13 & 1 & 1 \\
\hline Thailand & Asia & Mainland Southeast Asia & 28 & 8 & 6 \\
\hline The Gambia & Africa & West Africa & 0 & 0 & 0 \\
\hline The Netherlands & Europe & Northwest Europe & 123 & 197 & 192 \\
\hline Timor-Leste & Asia & Maritime Southeast Asia & 0 & 0 & 0 \\
\hline Togo & Africa & West Africa & 0 & 0 & 0 \\
\hline Tonga & Oceania & Polynesia & 0 & 0 & 0 \\
\hline Trinidad \& Tobago & South America & Caribbean & 0 & 0 & 0 \\
\hline Tunisia & Africa & North Africa & 15 & 1 & 2 \\
\hline Turkey & Europe & Eastern Europe & 281 & 169 & 158 \\
\hline Turkmenistan & Asia & Central Asia & 0 & 0 & 0 \\
\hline Tuvalu & Oceania & Polynesia & 0 & 0 & 0 \\
\hline Uganda & Africa & East Africa & 12 & 3 & 6 \\
\hline Ukraine & Europe & Eastern Europe & 0 & 0 & 1 \\
\hline United Arab Emirates & Asia & Arabian Peninsula & 8 & 0 & 3 \\
\hline United Kingdom & Europe & British Isles & 237 & 127 & 273 \\
\hline United States & North America & & 4375 & 4200 & 3724 \\
\hline Uruguay & South America & Southeastern South America & 3 & 0 & 3 \\
\hline
\end{tabular}


TABLE 1. Summary of countries, with information regarding their geographic location and research output in three neurosurgical journals

\begin{tabular}{|c|c|c|c|c|c|}
\hline \multirow[b]{2}{*}{ Country } & \multirow[b]{2}{*}{ Continent } & \multirow[b]{2}{*}{ Region } & \multicolumn{3}{|c|}{ No. of Publications } \\
\hline & & & World Neurosurg & Neurosurg & J Neurosurg \\
\hline Uzbekistan & Asia & Central Asia & 1 & 0 & 0 \\
\hline Vanuatu & Oceania & Melanesia & 0 & 0 & 0 \\
\hline Venezuela & South America & Northern South America & 1 & 1 & 0 \\
\hline Vietnam & Asia & Indochina Peninsula & 6 & 0 & 3 \\
\hline Yemen & Asia & Arabian Peninsula & 0 & 0 & 0 \\
\hline Zambia & Africa & South-Central Africa & 0 & 0 & 0 \\
\hline Zimbabwe & Africa & South Africa & 2 & 0 & 0 \\
\hline
\end{tabular}

J Neurosurg = Journal of Neurosurgery; Neurosurg = Neurosurgery; World Neurosurg = World Neurosurgery.

The table is organized alphabetically by country.

it was not required as a component of training. The average number of publications in World Neurosurgery, Journal of Neurosurgery, and Neurosurgery for each category of requirements is summarized in Fig. 1. Surprisingly, we observed a trend of increased average research productivity with the absence of designated research requirements. Similar results have been observed in a previous bibliometric analysis of research productivity among specific neurosurgical residency programs within the United States $^{19}$ and will be discussed later. The heterogeneity of our results, especially the variance in research productivity among nations, unfortunately prevented statistical analysis of the relationship between research requirements and national research productivity. In addition, when we averaged publications in each journal among all countries, we found that World Neurosurgery had the highest number of publications $(69.3$, range $0-4375$ vs 52.7 , range $0-4200$ and 53.4, range 0-3724 for Neurosurgery and Journal of Neurosurgery, respectively). This finding is consistent with the mission of World Neurosurgery to provide an "international forum" and "two-way dialogue" for neurosurgeons and those caring for neurosurgical patients. ${ }^{10}$ Once again, statistical analysis was not possible because of the high heterogeneity of the data. The breakdown of research productivity in these journals across continents is summarized in Fig. 2. Publication in these journals appears biased toward developed countries in North America, Europe, and Asia. Overall, we have been able to obtain and report interesting descriptive data regarding the types of research mandates imposed during training and/or prior to board certification via a global review of training curricula and requirements.

\section{Discussion}

The goal of our study was to identify and describe research requirements during neurosurgical training and/or prior to board certification across the globe. In addition, we sought to quantify research output across various nations via publication in 3 of the most prominent journals in the field: World Neurosurgery, Neurosurgery, and Journal of Neurosurgery. Notably, specific research requirements were identified in countries in Africa, Asia, Europe, and
Oceania. Countries for which requirement information was available in North America and South America identified no mandatory research requirements. We did not find a positive correlation between the presence of a research requirement and research output, as may have been intuited. In fact, our rudimentary analysis suggested that countries lacking research requirements had higher rates of research productivity, although this analysis did not account for other factors such as density of neurosurgeons, size, etc. A similar result was shown in a recent analysis of American residency program research output, in which mandated research did not correlate with improved productivity. ${ }^{19}$ Authors of that study postulated that this finding may be attributable to a culture in which research is informally established in many of the high-output institutions, thereby making a requirement unnecessary. However, for programs seeking to establish the culture, such a requirement may be useful.

While some countries detail explicit requirements for research experience during neurosurgical training, such as a minimum number of publications or presentations, others make broad recommendations or requirements, with individual programs then determining their own explicit guidelines. This is characteristic of the United States, in which the American Board of Neurological Surgery encourages resident involvement in research, and individual programs make subsequent decisions about the requirement for, and the timing of, research within the training program. ${ }^{3}$ We discuss below the research requirements of selected countries across the continents mentioned above, with a focus on the types of requirements and the timing of research involvement during training, as available.

\section{Africa}

African countries specifying research requirements spanned the continent in terms of regional representation; however, the majority fell within Eastern, Central, and Southern Africa in territories under the purview of the COSECSA, which has been instrumental in the development and standardization of surgical training curricula in a variety of surgical subspecialties, including neurosurgery, throughout Sub-Saharan Africa. COSECSA currently represents 14 countries, ${ }^{7}$ although according to the most re- 
TABLE 2. Summary of research requirements and output for countries

\begin{tabular}{|c|c|c|c|c|c|c|c|c|}
\hline \multirow[b]{2}{*}{ Country } & \multirow[b]{2}{*}{ Continent } & \multirow[b]{2}{*}{ Region } & \multirow[b]{2}{*}{$\begin{array}{l}\text { Requirement } \\
\text { Type }\end{array}$} & \multirow[b]{2}{*}{ Requirement(s) or Comment(s) } & \multirow[b]{2}{*}{$\begin{array}{c}\text { Requiring or } \\
\text { Reporting Agency }\end{array}$} & \multicolumn{3}{|c|}{ No. of Publications } \\
\hline & & & & & & $\begin{array}{c}\text { World } \\
\text { Neurosurg }\end{array}$ & Neurosurg & $\begin{array}{c}J \\
\text { Neurosurg }\end{array}$ \\
\hline Botswana & Africa & South Africa & Specific & Complete dissertation & COSECSA & 0 & 0 & 0 \\
\hline Burundi & Africa & East Africa & Specific & Complete dissertation & COSECSA & 0 & 0 & 0 \\
\hline Egypt & Africa & $\begin{array}{c}\text { Northeast } \\
\text { Africa }\end{array}$ & Specific & $\begin{array}{l}\text { Multiple degrees available to neu- } \\
\text { rosurgeons, } 1 \text { pathway requires } \\
\text { thesis defense }\end{array}$ & $\begin{array}{l}\text { Egyptian Society } \\
\text { of Neurological } \\
\text { Surgeons }\end{array}$ & 92 & 19 & 34 \\
\hline Ethiopia & Africa & East Africa & Specific & Complete thesis & COSECSA & 13 & 0 & 0 \\
\hline Ghana & Africa & West Africa & Specific & Complete dissertation & $\begin{array}{l}\text { Ghana College } \\
\text { of Physicians \& } \\
\text { Surgeons }\end{array}$ & 4 & 0 & 0 \\
\hline Kenya & Africa & East Africa & Specific & Complete dissertation & COSECSA & 15 & 0 & 0 \\
\hline Malawi & Africa & $\begin{array}{l}\text { Southeast } \\
\text { Africa }\end{array}$ & Specific & Complete dissertation & COSECSA & 2 & 0 & 0 \\
\hline Morocco & Africa & North Africa & Specific & $\begin{array}{l}\text { Unspecified no. publications \& } \\
\text { presentations }\end{array}$ & $\begin{array}{l}\text { WFNS Rabat Refer- } \\
\text { ence Center }\end{array}$ & 22 & 6 & 3 \\
\hline $\begin{array}{l}\text { Mozam- } \\
\text { bique }\end{array}$ & Africa & $\begin{array}{c}\text { Southeast } \\
\text { Africa }\end{array}$ & Specific & Complete dissertation & COSECSA & 2 & 0 & 0 \\
\hline Namibia & Africa & South Africa & Specific & Complete dissertation & COSECSA & 0 & 0 & 0 \\
\hline Nigeria & Africa & West Africa & Specific & Complete dissertation & $\begin{array}{l}\text { West African College } \\
\text { of Surgeon }\end{array}$ & 24 & 2 & 5 \\
\hline Rwanda & Africa & Central Africa & Specific & Complete dissertation & COSECSA & 6 & 0 & 0 \\
\hline $\begin{array}{l}\text { South } \\
\quad \text { Sudan }\end{array}$ & Africa & $\begin{array}{l}\text { East-Central } \\
\text { Africa }\end{array}$ & Specific & Complete dissertation & COSECSA & 0 & 0 & 0 \\
\hline Sudan & Africa & $\begin{array}{c}\text { Northeast } \\
\text { Africa }\end{array}$ & Specific & Complete dissertation & COSECSA & 3 & 0 & 0 \\
\hline Tanzania & Africa & East Africa & Specific & Complete dissertation & COSECSA & 13 & 1 & 1 \\
\hline Uganda & Africa & East Africa & Specific & Complete dissertation & COSECSA & 12 & 3 & 6 \\
\hline Zambia & Africa & $\begin{array}{l}\text { South-Cen- } \\
\text { tral Africa }\end{array}$ & Specific & Complete thesis \& 1 publication & $\begin{array}{l}\text { School of Medicine, } \\
\text { University of Zam- } \\
\text { bia; COSECSA }\end{array}$ & 0 & 0 & 0 \\
\hline Zimbabwe & Africa & South Africa & Specific & Complete dissertation & COSECSA & 2 & 0 & 0 \\
\hline Bahrain & Asia & Persian Gulf & Specific & Publish 1 article & ABNPE & 0 & 0 & 0 \\
\hline China & Asia & East Asia & None & $\begin{array}{l}\text { Neurosurgical training standard- } \\
\text { ized nationwide in 2015; will not } \\
\text { be fully implemented till 2020; } \\
\text { new program does not appear } \\
\text { to have specific research } \\
\text { requirements or provide dedi- } \\
\text { cated research time }\end{array}$ & $\begin{array}{l}\text { Chinese Congress } \\
\text { of Neurological } \\
\text { Surgeons }\end{array}$ & 1946 & 235 & 336 \\
\hline India & Asia & South Asia & Specific & Complete dissertation & $\begin{array}{l}\text { Medical Council of } \\
\text { India }\end{array}$ & 448 & 121 & 136 \\
\hline Iraq & Asia & West Asia & Specific & Publish 1 article & ABNPE & 6 & 1 & 0 \\
\hline Japan & Asia & Eastern Asia & Specific & $>1$ presentation \& 1 publication & $\begin{array}{l}\text { Japan Neurosurgical } \\
\text { Society }\end{array}$ & 1108 & 1230 & 1685 \\
\hline Jordan & Asia & Western Asia & Specific & Publish 1 article & ABNPE & 7 & 2 & 2 \\
\hline Kuwait & Asia & $\begin{array}{l}\text { Arabian } \\
\text { Peninsula }\end{array}$ & Specific & $\begin{array}{l}\text { Required to take } 3 \text { mos dedicated } \\
\text { research time }\end{array}$ & $\begin{array}{l}\text { Ministry of Health, } \\
\text { Kuwait }\end{array}$ & 8 & 2 & 2 \\
\hline Lebanon & Asia & Western Asia & Specific & $\begin{array}{l}\text { Publish } 1 \text { peer-reviewed article \& } \\
1 \text { presentation }\end{array}$ & ABNPE & 48 & 56 & 47 \\
\hline Malaysia & Asia & $\begin{array}{l}\text { Maritime } \\
\text { Southeast } \\
\text { Asia }\end{array}$ & Specific & Complete thesis & $\begin{array}{l}\text { Neurosurgical Asso- } \\
\text { ciation of Malaysia }\end{array}$ & 10 & 4 & 4 \\
\hline
\end{tabular}


» CONTINUED FROM PAGE 8

TABLE 2. Summary of research requirements and output for countries

\begin{tabular}{|c|c|c|c|c|c|c|c|c|}
\hline \multirow[b]{2}{*}{ Country } & \multirow[b]{2}{*}{ Continent } & \multirow[b]{2}{*}{ Region } & \multirow[b]{2}{*}{$\begin{array}{l}\text { Requirement } \\
\text { Type }\end{array}$} & \multirow[b]{2}{*}{ Requirement(s) or Comment(s) } & \multirow[b]{2}{*}{$\begin{array}{c}\text { Requiring or } \\
\text { Reporting Agency }\end{array}$} & \multicolumn{3}{|c|}{ No. of Publications } \\
\hline & & & & & & $\begin{array}{l}\text { World } \\
\text { Neurosurg }\end{array}$ & Neurosurg & $\begin{array}{c}J \\
\text { Neurosurg }\end{array}$ \\
\hline Qatar & Asia & $\begin{array}{c}\text { Arabian } \\
\text { Peninsula }\end{array}$ & Specific & Publish 1 article & ABNPE & 5 & 0 & 2 \\
\hline $\begin{array}{l}\text { Saudi } \\
\text { Arabia }\end{array}$ & Asia & $\begin{array}{c}\text { Arabian } \\
\text { Peninsula }\end{array}$ & Specific & Publish 1 article & ABNPE & 71 & 15 & 22 \\
\hline $\begin{array}{l}\text { Syrian } \\
\text { Arab } \\
\text { Republic }\end{array}$ & Asia & West Asia & Specific & Publish 1 article & ABNPE & 1 & 0 & 0 \\
\hline Albania & Europe & $\begin{array}{c}\text { Balkan } \\
\text { Peninsula }\end{array}$ & Specific & Present research thesis & $\begin{array}{l}\text { School of Neurosur- } \\
\text { gery }\end{array}$ & 4 & 0 & 3 \\
\hline Croatia & Europe & $\begin{array}{c}\text { Balkan } \\
\text { Peninsula }\end{array}$ & Partial & $\begin{array}{l}\text { NDA on national requirements, } \\
\text { but JRAAC accredited } 2 \text { neuro- } \\
\text { surgical programs in Croatia, \& } \\
\text { per JRAAC, residents must be } \\
\text { trained on research methodolo- } \\
\text { gy, but no specific requirements }\end{array}$ & JRAAC & 12 & 3 & 8 \\
\hline Denmark & Europe & Scandinavia & Partial & $\begin{array}{l}\text { NDA on national requirements, } \\
\text { but JRAAC accredited } 1 \\
\text { neurosurgical program in Den- } \\
\text { mark, \& per JRAAC, residents } \\
\text { must be trained on research } \\
\text { methodology, but no specific } \\
\text { requirements }\end{array}$ & JRAAC & 25 & 31 & 36 \\
\hline Germany & Europe & $\begin{array}{l}\text { Western } \\
\text { Europe }\end{array}$ & Partial & $\begin{array}{l}\text { NDA on national requirements, } \\
\text { but JRAAC accredited } 4 \text { major } \\
\text { neurosurgical programs in Ger- } \\
\text { many, \& per JRAAC, residents } \\
\text { must be trained on research } \\
\text { methodology, but no specific } \\
\text { requirements }\end{array}$ & JRAAC & 586 & 754 & 644 \\
\hline Ireland & Europe & British Isles & Specific & $\begin{array}{l}\text { Publish } 1 \text { peer-reviewed article \& } \\
1 \text { presentation }\end{array}$ & $\begin{array}{l}\text { Joint Committee on } \\
\text { Surgical Training }\end{array}$ & 22 & 19 & 14 \\
\hline
\end{tabular}




\begin{tabular}{|c|c|c|c|c|c|c|c|c|}
\hline \multirow[b]{2}{*}{ Country } & \multirow[b]{2}{*}{ Continent } & \multirow[b]{2}{*}{ Region } & \multirow[b]{2}{*}{$\begin{array}{l}\text { Requirement } \\
\text { Type }\end{array}$} & \multirow[b]{2}{*}{ Requirement(s) or Comment(s) } & \multirow[b]{2}{*}{$\begin{array}{c}\text { Requiring or } \\
\text { Reporting Agency }\end{array}$} & \multicolumn{3}{|c|}{ No. of Publications } \\
\hline & & & & & & $\begin{array}{l}\text { World } \\
\text { Neurosurg }\end{array}$ & Neurosurg & $\begin{array}{c}J \\
\text { Neurosurg }\end{array}$ \\
\hline Lithuania & Europe & $\begin{array}{l}\text { Northern } \\
\text { Europe }\end{array}$ & Partial & $\begin{array}{l}\text { NDA on national requirements, } \\
\text { but JRAAC accredited } 1 \\
\text { neurosurgical program in Lithu- } \\
\text { ania, \& per JRAAC, residents } \\
\text { must be trained on research } \\
\text { methodology, but no specific } \\
\text { requirements }\end{array}$ & JRAAC & 4 & 2 & 1 \\
\hline Malta & Europe & $\begin{array}{l}\text { Mediterra- } \\
\text { nean Basin }\end{array}$ & Specific & $\begin{array}{l}\text { Publish } 2 \text { articles \& conduct } 2 \\
\text { clinical audits }\end{array}$ & $\begin{array}{l}\text { Specialist Accredita- } \\
\text { tion Committee }\end{array}$ & 0 & 0 & 0 \\
\hline Poland & Europe & $\begin{array}{l}\text { Central } \\
\text { Europe }\end{array}$ & Partial & $\begin{array}{l}\text { NDA on national requirements, } \\
\text { but JRAAC accredited } 1 \text { neuro- } \\
\text { surgical program in Poland, \& } \\
\text { per JRAAC, residents must be } \\
\text { trained on research methodolo- } \\
\text { gy, but no specific requirements }\end{array}$ & JRAAC & 36 & 9 & 16 \\
\hline Portugal & Europe & $\begin{array}{l}\text { Iberian } \\
\text { Peninsula }\end{array}$ & Partial & $\begin{array}{l}\text { NDA on national requirements, } \\
\text { but JRAAC accredited } 1 \text { neuro- } \\
\text { surgical program in Portugal, \& } \\
\text { per JRAAC, residents must be } \\
\text { trained on research methodolo- } \\
\text { gy, but no specific requirements }\end{array}$ & JRAAC & 17 & 15 & 7 \\
\hline $\begin{array}{l}\text { Russian } \\
\text { Federa- } \\
\text { tion }\end{array}$ & Europe & $\begin{array}{l}\text { Eastern } \\
\text { Europe }\end{array}$ & None & NA & NA & 61 & 5 & 14 \\
\hline Spain & Europe & $\begin{array}{l}\text { Southwestern } \\
\text { Europe }\end{array}$ & Specific & $\begin{array}{l}\text { Training on research methodol- } \\
\text { ogy \& involvement in research } \\
\text { project resulting in presenta- } \\
\text { tion/publication }\end{array}$ & $\begin{array}{l}\text { Comisión Nacional } \\
\text { de Neurocirugía }\end{array}$ & 169 & 110 & 144 \\
\hline Turkey & Europe & $\begin{array}{l}\text { Eastern } \\
\text { Europe }\end{array}$ & Specific & Complete thesis & $\begin{array}{l}\text { Turkish Neurosurgi- } \\
\text { cal Society }\end{array}$ & 281 & 169 & 158 \\
\hline $\begin{array}{l}\text { United } \\
\text { King- } \\
\text { dom }\end{array}$ & Europe & British Isles & Specific & $\begin{array}{l}\text { Research encouraged but no } \\
\text { specific requirements }\end{array}$ & $\begin{array}{l}\text { Joint Committee on } \\
\text { Surgical Training }\end{array}$ & 237 & 127 & 273 \\
\hline Canada & $\begin{array}{l}\text { North } \\
\text { America }\end{array}$ & & None & NA & $\begin{array}{l}\text { Royal College of } \\
\text { Physicians \& Sur- } \\
\text { geons of Canada }\end{array}$ & 342 & 570 & 682 \\
\hline Haiti & $\begin{array}{c}\text { North } \\
\text { America }\end{array}$ & Caribbean & None & NA & $\begin{array}{l}\text { Haitian Ministry of } \\
\text { Health, Hospital } \\
\text { Bernard-Mevs/Proj- } \\
\text { ect Medishare }\end{array}$ & 5 & 0 & 1 \\
\hline $\begin{array}{l}\text { United } \\
\text { States }\end{array}$ & $\begin{array}{c}\text { North } \\
\text { America }\end{array}$ & & None & NA & $\begin{array}{l}\text { American Board } \\
\text { of Neurological } \\
\text { Surgeons }\end{array}$ & 4375 & 4200 & 3724 \\
\hline Australia & Oceania & & Specific & 1 presentation \& 1 publication & $\begin{array}{l}\text { Neurosurgical Soci- } \\
\text { ety of Australasia }\end{array}$ & 140 & 140 & 117 \\
\hline $\begin{array}{l}\text { New Zea- } \\
\text { land }\end{array}$ & Oceania & Polynesia & Specific & Publish 1 article \& 1 presentation & $\begin{array}{l}\text { Neurosurgical Soci- } \\
\text { ety of Australasia }\end{array}$ & 18 & 14 & 9 \\
\hline Colombia & $\begin{array}{l}\text { South } \\
\text { America }\end{array}$ & $\begin{array}{l}\text { Northern } \\
\text { South } \\
\text { America }\end{array}$ & None & NA & $\begin{array}{l}\text { No centralized certi- } \\
\text { fication system }\end{array}$ & 35 & 14 & 8 \\
\hline
\end{tabular}

ABNPE = Arab Board of Neurosurgical Programs and Examinations; COSECSA = College of Surgeons of East, Central and Southern Africa; JRAAC = Joint Residency Advisory and Accreditation Committee; NA = not applicable; NDA = no data available; WFNS Rabat Reference Center = WFNS Rabat Reference Center for Training Young African Neurosurgeons. 


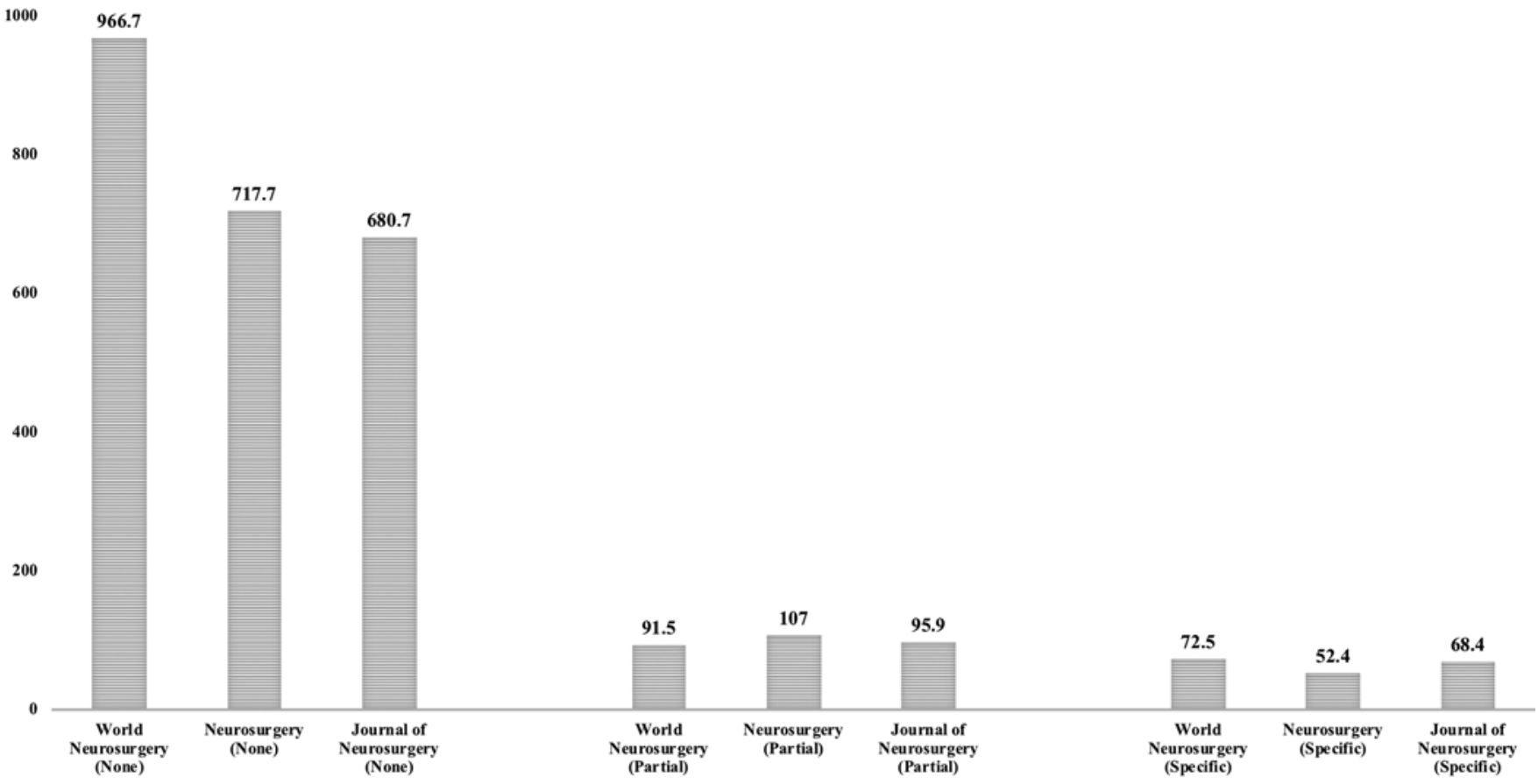

FIG. 1. Quantification of publications in World Neurosurgery, Neurosurgery, and Journal of Neurosurgery among countries detailing no research requirements, partial research requirements, or specific research requirements.

cently available data, accredited programs are only present in Kenya, Uganda, Tanzania, Ethiopia, Zambia, Rwanda, and Malawi. ${ }^{14,31}$ In the training guidelines established by COSECSA, trainees who have earned a doctorate in medicine are registered as members of the COS (MCS) after they complete 2 years of training and subsequently complete 4 years of training in neurosurgery, culminating in the Fellow of the COS (FCS) certification. ${ }^{6}$ Participation in research projects during this training period is expected, including completion of a dissertation ${ }^{14}$ and prepara- tion of a presentation for an international surgical conference. ${ }^{6}$ Additionally, 1 non-thesis research paper must be submitted at least 3 months prior to sitting for the oral/ clinical FCS examination. ${ }^{6}$ Individual programs within Africa have also implemented research requirements, including the newly developed University of Zambia School of Medicine Neurosurgery Training Program, developed in partnership with the University of Cambridge. This program, spanning 5 years and 3 stages (initial, intermediate, and final), requires registrars to complete a thesis for the

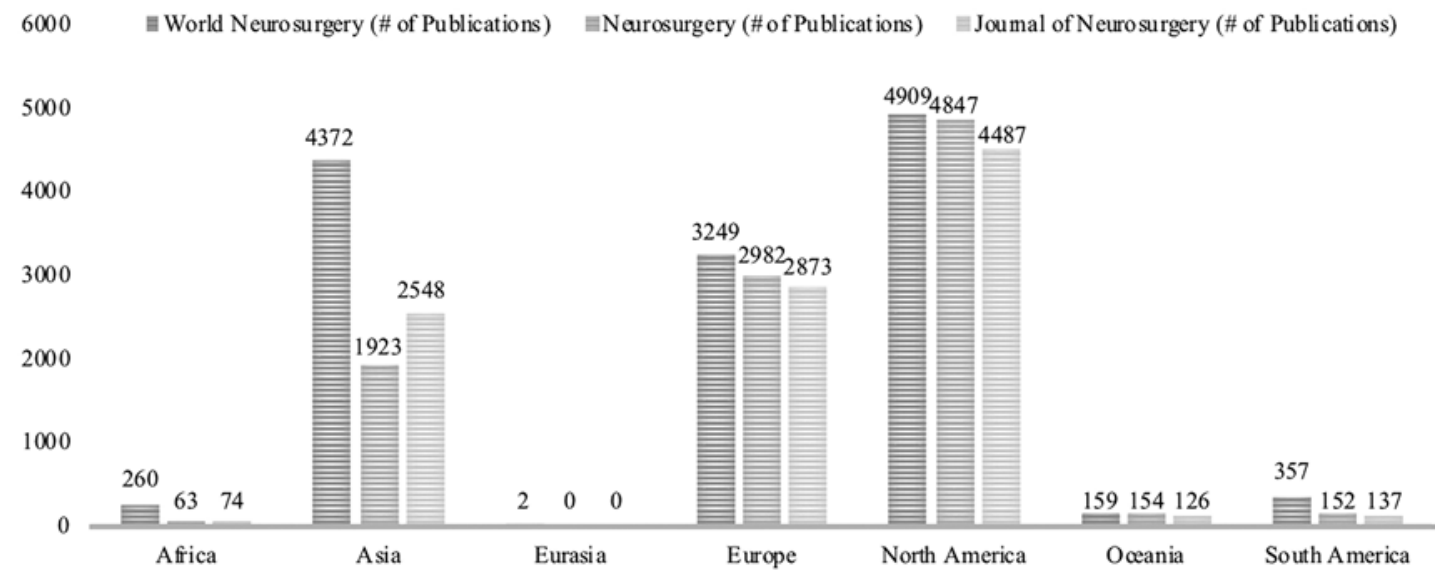

FIG. 2. Quantification of publications in World Neurosurgery, Neurosurgery, and Journal of Neurosurgery across continents. 
Master of Medicine degree, with research beginning in the first 2 years and completed in the 3rd or 4th years. Publication of at least 1 peer-reviewed article is required prior to progression to the chief registrar year. During this final year, registrars take on additional scholarly responsibility including delivering student lectures and running morbidity and mortality conferences. ${ }^{30}$

\section{Asia}

Once again, the specification of research requirements traverses regional boundaries across the Asian continent. Within the Middle East and Gulf region, neurosurgical training and certification is overseen by the Arab Board of Neurosurgical Programs and Examinations and includes the countries of Iraq, Jordan, Bahrain, Saudi Arabia, Lebanon, Qatar, and Syria. In the 5th or 6th years of the training program, trainees take the final examination for certification including written, clinical, and oral portions. Prior to this examination, trainees must complete and publish 1 research project, as well as maintain a log of attendance at scientific sessions. ${ }^{4,14}$ Kuwait is one of the few countries whose Ministry of Health mandates the completion of a dedicated research period during neurosurgical training. This dedicated time occurs during the 3rd year of a 5-year program,$^{20}$ resembling the timing of the research year that is typical of many programs within the United States. Japan is one of the most productive countries in neurosurgical research that utilizes a research requirement. The Japan Neurosurgical Society requires an individual to present at an academic conference more than once and write research papers, at least one of which must be accepted into a peer-reviewed journal to obtain eligibility for the board certification examination. Additionally, recertification is contingent upon continued scholarly work in the form of academic presentations and/or publications. ${ }^{16}$ According to our analysis, Japan ranks just second to the United States in research output in the American literature, suggesting that its requirements place an appropriate emphasis on lifelong scholarship, which translates into exceptional productivity. Notably, a similarly industrialized nation, China, bears no research requirement in its newly developed standardized training program, which is set for nationwide adoption by 2020 . But a lack of flexibility in the training schedule, limiting the time for research and/ or enfolded fellowships, is considered a limitation of this program. ${ }^{32}$ While China ranks highly at present in terms of research output, the impact of the program's design may perturb this. Finally, in Singapore, while no specific requirements for research involvement exist, programs are required to teach principles of research within the training curriculum. Residents are encouraged to participate in scholarly activity, although this is undefined, and sponsoring institutions are encouraged to provide adequate resources for such involvement. ${ }^{29}$

\section{Europe}

The JRAAC is the central monitoring body for the field of neurosurgery within Europe and is a joint committee between the European Association of Neurosurgical Societies (EANS) and the European Union of Medical Specialists (UEMS). There are currently 16 JRAAC-accred- ited programs in Turkey, Germany, Czech Republic, Denmark, Lithuania, Poland, Spain, Portugal, and Croatia. ${ }^{11}$ Guidelines released from the UEMS in 2015 recommend that neurosurgical training programs provide training in research methodology, as well as the opportunity for protected research time during the 6 -year training program. ${ }^{12}$ However, these recommendations do not represent explicit requirements for compulsory participation in research as a trainee. Despite the lack of requirements by this multinational organization, individual nations within Europe do maintain prerequisites for research participation during training. For example, in the United Kingdom (UK) and Republic of Ireland, where neurosurgical training is overseen by the Joint Committee on Surgical Training, research requirements are quite extensive: trainees must publish 1 peer-reviewed paper covering a laboratory experiment and a case series or systematic audit, as well as deliver 2 verbal presentations at national or international conferences, with attendance at a minimum of 4 conferences in total during training. ${ }^{17}$ Furthermore, in Albania, presentation of one's research thesis is, in fact, a component of the final board-certifying examination..$^{27}$

\section{Oceania}

Neurosurgical training in Australia and New Zealand is overseen by the Neurosurgical Society of Australasia, an organization founded in 2014, which currently boasts a membership of $95 \%$ of neurosurgeons within the 2 countries. ${ }^{25}$ The neurosurgical training program guided by the society involves 3 phases: basic, constituting 1-2 years; intermediate, constituting 2-3 years; and advanced, constituting 1-3 years. Research requirements are primarily restricted to the intermediate years including participation in the Critical Literature Evaluation and Research course and completion, presentation, and publication of an approved research project. In fact, prior to 2012, all trainees in neurosurgical training programs were required to complete a compulsory research year during the 4 th year of training. ${ }^{26}$

\section{Barriers to Trainee Research Involvement}

Studies identifying barriers to resident/trainee research involvement have been conducted in several countries, identifying results similar to those within the United States: a lack of protected time for both trainees and faculty, a lack of mentorship, and unfamiliarity with research methodologies continue to be reported as the greatest obstacles. ${ }^{5,22}$ But research requirements that are instituted in environments conducive to research and offering the necessary training, resources, and protected time are likely to be more fruitful than similar requirements without the necessary support. International collaboration may provide an important avenue to enhance the research involvement of trainees from institutions without a strong research infrastructure, especially those within low- and middle-income countries. Various models for collaboration exist, often with the primary goal of enhancing clinical productivity within developing countries, although opportunities for involvement in research exist as well. One of the most common models involves integration of a visiting neurosurgeon within the department of a host institution for a short- or long-term fellowship, during which the 
visiting fellow trains in clinical and operative care while also participating in clinical research projects. ${ }^{2}$ Trainees can also seek involvement in research collaboratives by partnering their home countries with large international institutions. For example, the UK-based Global Health Research Group on Neurotrauma is conducting a multinational research project to identify optimal strategies for the management of patients with traumatic brain injury via partnership with countries throughout South America, Asia, and Africa. ${ }^{23}$

\section{Study Limitations}

This analysis represents a review of trainee research requirements across the globe via internet query of guidelines produced by certifying and/or accrediting bodies, as well as regional research productivity via advanced search of the PubMed database. We recognize that our study is limited in scope because of its third-person analysis of published requirements, rather than direct survey of individuals from the various nations. While we can comment on the presence or absence of documented guidelines or requirements, we cannot determine the way in which these requirements are enforced, nor how they are perceived by trainees and faculty. Additionally, our study is inherently biased toward English-language literature based on the restriction of our literature search to the PubMed database. Publication within the major peer-reviewed journals of the field is considered a gold standard for the dissemination of research. However, barriers to publication do exist, such as language differences and financial limitations. Various initiatives have been undertaken by neurosurgical publishers to promote access to neurosurgical literature by stakeholders across the world. As an example, the Neurosurgery Speaks program, undertaken by Neurosurgery, provides written and oral abstracts for dozens of publications translated into 10 different languages by native speakers. ${ }^{8}$ We also cannot fail to acknowledge the importance of international scholarly contributions outside of the peerreviewed literature, including social media posts and digital teaching. The website Neurosurgical.TV (https://www. neurosurgical.tv/) provides a forum for hosting video conferences by neurosurgeons across the globe and is broadly shared across neurosurgery pages on social media platforms such as Facebook and Twitter. While these are not a substitute for the peer-review process, they are important modalities for improving international dissemination of neurosurgical research and knowledge. We anticipate that as social media continues to rise in prominence within neurosurgery and the field of medicine as a whole, tools for the assessment of scholarly productivity will adapt to account for such contributions.

\section{Recommendations and Future Directions}

Clinical experience must remain the focus of neurosurgical training to maintain the most appropriate care for patients. However, the importance of research and scholarship in establishing training programs and evolving care cannot be understated. Protected research time is common in training programs throughout developed countries, but is often reliant on an established research infrastructure. Thus, partnerships permitting integration of local train- ees into the scholarly activities of an outside institution may circumvent a lack of resources in less-developed programs..$^{28}$ Institutions in developed countries seeking to aid in global surgical efforts can effect some of the greatest changes by assisting in the development of local training programs, as has been accomplished via Duke University and Mulago Hospital in Uganda. ${ }^{13}$ Such efforts should include training in research methodologies and collaborative projects to characterize aspects of neurosurgical care in developing countries and assess the impact of any interventions that are instituted. Finally, we believe instituting a requirement can help establish research involvement in the culture of an institution and/or nation. Future prospective research in the form of surveys administered to trainees and faculty across the globe would provide a more direct assessment of the requirements and understanding of individuals' personal experiences. We believe it is critical for such perspectives to be collected to inform efforts targeted at augmenting research productivity.

\section{Conclusions}

Overall, we found that research requirements during training or prior to board certification are common in neurosurgical programs across the globe as an incentive to contribute novel knowledge to the field while maintaining research productivity and academic standing. These requirements vary in intensity and timing during training, ranging from exposure to research methodology or critical literature evaluation to completion of a thesis and publication in a peer-reviewed journal. While our analysis is quite limited, we failed to demonstrate a correspondence between the presence of requirements and research output. We hope that this study will provide a set of novel preliminary data through which the global neurosurgery community can begin to systematically assess the importance of research participation throughout the spectrum of neurosurgical training.

\section{Acknowledgments}

We would like to acknowledge the incredible work of all neurosurgical educators who are working tirelessly to ensure the continued growth of our specialty and the delivery of optimal care to our patients across the globe.

\section{References}

1. Accreditation Council for Graduate Medical Education: Program Requirements for Graduate Medical Education in Neurological Surgery. (https://www. acgme.org/portals/0/pfassets/programrequirements/160_ neurologicalsurgery_2019.pdf?ver=2019-06-20-114304-737) [Accessed January 20, 2020]

2. Almeida JP, Velásquez C, Karekezi C, Marigil M, Hodaie M, Rutka JT, et al: Global neurosurgery: models for international surgical education and collaboration at one university. Neurosurg Focus 45(4):E5, 2018

3. American Board of Neurological Surgery: ABNS Training Requirements. (https://www.abns.org/wp-content/ uploads/2019/08/abns-training-requirements.pdf) [Accessed January 20, 2020]

4. Arab Board of Health Specializations: Neurosurgery Logbook Summary, Febuary 2018. (http://arab-board.org/sites/ 
default/files/neurosurgery\%20logbook\%202018.pdf) [Accessed January 20, 2020]

5. Clancy AA, Posner G: Attitudes toward research during residency: a survey of Canadian residents in obstetrics and gynecology. J Surg Educ 72:836-843, 2015

6. College of Surgeons of East, Central, and South Africa: Training Curriculum: Fellowship in General Surgery 2019. (http://www.cosecsa.org/sites/default/files/fcs\%20 gs\%20curriculum\%20final.pdf) [Accessed January 20, 2020]

7. College of Surgeons of East, Central, and South Africa: What is COSECSA? COSECSA.org (http://www.cosecsa.org/ about/what-cosecsa) [Accessed January 20, 2020]

8. Congress of Neurological Surgeons: Neurosurgery Speaks! Neurosurgery. (https://academic.oup.com/neurosurgery/ pages/neurosurgeryspeaks) [Accessed January 20, 2020]

9. Elkbuli A, Narvel RI, Zajd S, Hai S, McKenney M, Boneva D: Factors affecting research productivity of burn surgeons: results from a survey of American Burn Association members. J Burn Care Res [epub ahead of print], 2019

10. Elsevier: World Neurosurgery. Elsevier.com (https://www. journals.elsevier.com/world-neurosurgery) [Accessed January 20, 2020]

11. European Association of Neurosurgical Societies: JRAAC accredited units. EANS.org (https://www.eans.org/page/ jraac-units) [Accessed January 20, 2020]

12. European Union of Medical Specialists Section of Neurosurgery: European Training Requirements for the Specialty of Neurosurgery. (https://www.uems.eu/_data/ assets/pdf_file/0007/44449/uems-2015.34-european-trainingrequirements-in-neurosurgery.pdf) [Accessed January 20, 2020]

13. Fuller A, Tran T, Muhumuza M, Haglund MM: Building neurosurgical capacity in low and middle income countries. eNeurologicalSci 3:1-6, 2015

14. Gasco J, Barber SM, McCutcheon IE, Black PM: Neurosurgery certification in member societies of the WFNS: Africa and the Middle East. World Neurosurg 76:18-27, 54-56, 2011

15. Gasco J, Barber SM, McCutcheon IE, Black PM: Neurosurgery certification in member societies of the WFNS: global overview. World Neurosurg 76:231-238, 2011

16. Japan Neurosurgical Society: Regulations for the Board Certification System. (http://jns.umin.ac.jp/english/boardregulations) [Accessed January 20, 2020]

17. Joint Committee on Surgical Training: Certification guidelines for neurosurgery. (https://www.jcst.org/-/media/files/ jcst/certification-guidelines-and-checklists/certificationguidelines--ns-2017-final.pdf) [Accessed January 20, 2020]

18. Kashkoush A, Prabhu AV, Tonetti D, Agarwal N: The Neurosurgery Match: a bibliometric analysis of 206 first-year residents. World Neurosurg 105:341-347, 2017

19. Khan NR, Saad H, Oravec CS, Norrdahl SP, Fraser B, Wallace $\mathrm{D}$, et al: An analysis of publication productivity during residency for 1506 neurosurgical residents and 117 residency departments in North America. Neurosurgery 84:857-867, 2019

20. Kuwait Ministry of Health: Kuwait Institute for Medical Specialization Neurosurgery Residency Program. (http:// kims.org.kw/pge/uploads/pdf/pdf-1252469562.pdf) [Accessed January 20, 2020]

21. Mansi A, Karam WN, Chaaban MR: Attitudes of residents and program directors towards research in otolaryngology residency. Ann Otol Rhinol Laryngol 128:28-35, 2019

22. Nair SC, Ibrahim H, Almarzoqi F, Alkhemeiri A, Sreedharan $\mathrm{J}$ : Addressing research barriers and facilitators in medical residency. J Family Med Prim Care 8:1145-1150, 2019
23. National Institute for Health Research: Global Health Research Group on Neurotrauma. Neurotrauma.world (http:// neurotrauma.world/about) [Accessed January 20, 2020]

24. National Resident Matching Program: Charting Outcomes in the Match: U.S. Allopathic Seniors. (https://www.nrmp. org/wp-content/uploads/2018/06/charting-outcomes-in-thematch-2018-seniors.pdf) [Accessed October 17, 2019]

25. Neurosurgical Society of Australasia: About us. NSA.org.au (https://nsa.org.au/nsa/about_us/nsa/) [Accessed January 20, 2020)

26. Neurosurgical Society of Australasia: Regulations for the Surgical Education and Training Program in Neurosurgery. (https://www.nsa.org.au/documents/neurosurgial\%20 training/regulations\%20training\%20program.pdf) [Accessed January 20, 2020]

27. Omerhodzic I, Tonge M, Matos B, Musabeliu E, Raspanti C, Ferdinandov D, et al: Neurosurgical training programme in selected European countries: from the young neurosurgeons' point of view. Turk Neurosurg 22:286-293, 2012

28. Servadei F, Rossini Z, Nicolosi F, Morselli C, Park KB: The role of neurosurgery in countries with limited facilities: facts and challenges. World Neurosurg 112:315-321, 2018

29. Singapore Ministry of Health: Specialists Accreditation Board Neurosurgery Residency Training Requirements. (https://www.healthprofessionals.gov.sg/docs/ librariesprovider9/downloads/neurosurgery-residencytraining-requirements-(as-@-14-sept-18).pdf) [Accessed January 20, 2020]

30. University of Zambia School of Medicine: Proposal for Neurosurgery Training Programme. (http://neurotrauma. world/wp-content/uploads/2018/07/proposal-forneurosurgery-training-programme_v2.pdf) [Accessed January 20,2020]

31. World Federation of Neurosurgical Societies: Criteria of The C-CNS-ECSAR WFNS Reference Center. WFNS.org (https://www.wfns.org/training-centers/37/) [Accessed January 20, 2020]

32. Xu T, Evins AI, Lin N, Chang J, Hu G, Hou L, et al: Neurosurgical postgraduate training in China: moving toward a national training standard. World Neurosurg 96:410-416, 2016

\section{Disclosures}

The authors report no conflict of interest concerning the materials or methods used in this study or the findings specified in this paper.

\section{Author Contributions}

Conception and design: Nanda, Rallo, Ashraf. Acquisition of data: Rallo, Ashraf, Jumah. Analysis and interpretation of data: Rallo, Ashraf, Gupta. Drafting the article: Rallo, Ashraf, Jumah, Gupta. Critically revising the article: Nanda, Rallo, Jumah, Gupta. Reviewed submitted version of manuscript: all authors. Approved the final version of the manuscript on behalf of all authors: Nanda. Statistical analysis: Rallo. Administrative/technical/material support: Nanda. Study supervision: Nanda, Gupta.

\section{Correspondence}

Anil Nanda: Rutgers Robert Wood Johnson Medical School, New Brunswick, NJ.an651@rwjms.rutgers.edu. 